\title{
Devant le marché...
}

Une note autour du texte de Franz Kafka : « Devant la loi »

\section{Roberto Baranzini}

\section{(2) OpenEdition}

\section{Journals}

Édition électronique

URL : http://journals.openedition.org/ress/466

DOI : $10.4000 /$ ress.466

ISSN : 1663-4446

Éditeur

Librairie Droz

\section{Édition imprimée}

Date de publication : 1 janvier 2006

Pagination : 103-108

ISBN : 2-600-00959-0

ISSN : 0048-8046

\section{Référence électronique}

Roberto Baranzini, « Devant le marché... », Revue européenne des sciences sociales [En ligne], XLIV-133 | 2006, mis en ligne le 10 novembre 2009, consulté le 01 mai 2019. URL : http:// journals.openedition.org/ress/466; DOI : 10.4000/ress.466 


\title{
Roberto BARANZINI
}

\section{DEVANT LE MARCHÉ... Une note autour du texte de Franz Kafka: «Devant la loi»}

On ne veut pas ici faire le constat, tout compte fait banal et facile, de la distance qui sépare la théorisation économique contemporaine des enjeux évoqués par Kafka, mais plutôt montrer comment un point central de la nouvelle «Devant la loi », qui a priori est tout à fait familier à la réflexion de l'économiste, ne peut plus être pensé par l'économiste*.

\section{UN POINT CENTRAL...}

L'angle d'approche que nous adoptons s'inspire fortement de l'article introductif de Pierre Livet et Gérard Timsit, notamment lorsqu'ils indiquent que

\begin{abstract}
...la nouvelle de Kafka donne à voir l'absurde des situations où nous nous trouvons placés, qui tient au refus ou à la dépossession du sens dont nous sommes victimes lorsque ce que nous ne comprenons pas se révèle pourtant déterminer notre destin - situations qui engendrent un malaise ou un mal-être, parce qu'elles sont duales. D'une part elles se proposent comme mettant en jeu ce qui règle nos interactions et donne à nos coordinations leurs repères, à nos litiges leurs aboutissements, d'autre part elles sont vécues comme ce qui méconnaît nos aspirations et dénie nos tentatives de donner sens et valeurs à nos interactions. (page ?? de cette revue)
\end{abstract}

Nous relevons ici deux éléments qui vont orienter cette discussion: la coordination et la connaissance des mécanismes de la coordination. Il s'agit de montrer que la réponse la plus récente et la plus utilisée par les économistes à la question de la connaissance des mécanismes de coordination de la part des acteurs, rend caduque la question même de la coordination.

\section{Coordination}

Pour chercher à traduire les préoccupations du texte de Kafka dans le domaine disciplinaire de l'économie, nous pouvons considérer le marché et les prix. Le marché me semble pouvoir assumer le rôle de l'institution et le prix celui de régulateur des actions. Dit autrement, le marché est considéré comme l'institution de coordination entre les choix des individus, une institution qui génère des règles

* Je remercie Juan Manuel Blanco pour son aide, Joanna Bauvert et Yves Steiner pour leur relecture. Il va de soi qu'ils ne sont pas responsables des erreurs éventuelles. 
qui sont les prix. /Régler/ signifie aussi 'mettre un terme à, conclure, résoudre définitivement, arrêter' et plus spécifiquement, 'payer'. D'ailleurs, on parle souvent du marché en tant qu'institution de régulation: les économistes qui considèrent le marché comme la seule instance de régulation légitime sont nombreux, raison pour laquelle ils appellent à la dérégulation, à l'abandon des autres instances possibles.

Si le marché fonctionne en tant que régulateur, le prix est à la fois le moyen par lequel l'offre et la demande s'ajustent et le résultat de cet ajustement. En d'autres termes, les prix sont les informations qui permettent aux volontés opposées des demandeurs et des offreurs de se coordonner et l'aboutissement de cette coordination.

Pour Léon Walras, la théorie de la détermination de la valeur d'échange de la richesse sociale relève d'une science naturelle puisque dans un régime de libre concurrence absolue, les prix s'imposent à la volonté des échangistes.

\begin{abstract}
Le blé vaut 24 F l'hectolitre. Remarquons d'abord que ce fait a le caractère d'un fait naturel. Cette valeur du blé en argent, ou ce prix du blé, ne résulte ni de la volonté du vendeur, ni de la volonté de l'acheteur, ni d'un accord entre les deux. Le vendeur voudrait bien vendre plus cher; il ne le peut, parce que le blé ne vaut pas plus, et que, s'il ne voulait vendre à ce prix, l'acheteur trouverait à côté de lui un certain nombre de vendeurs prêts à le faire. L'acheteur ne demanderait pas mieux que d'acheter à meilleur marché; cela lui est impossible, parce que le blé ne vaut pas moins, et que, s'il ne voulait acheter à ce prix, le vendeur trouverait à côté de lui un certain nombre d'acheteurs disposés à y consentir.
\end{abstract}

Le fait de la valeur d'échange prend donc, une fois établi, le caractère d'un fait naturel, naturel dans son origine, naturel dans sa manifestation et sa manière d'être. (Walras 1988, p. 50)

Léon Walras est considéré le fondateur de la théorie de l'équilibre général, le seul cadre (économique) pour juger de la coordination des actions décentralisées des agents. Est-ce qu'il existe un vecteur de prix tel que tous les marchés soient simultanément à l'équilibre? Ce vecteur est-il unique? Existent-ils des processus qui permettent d'atteindre cet équilibre? Voici les trois questions qui ont constitué le programme de recherche de la théorie de l'équilibre général pendant le $\mathrm{XX}^{\mathrm{e}}$ siècle ${ }^{1}$.

\title{
...QUI A ÉTÉ PENSÉ PAR L'ÉCONOMISTE...
}

D'une certaine façon, la question de la coordination des choix des acteurs a toujours concerné l'économie politique. La référence la plus connue est certainement celle de la 'main invisible' d'Adam Smith' .

Ils [les riches] ne consomment guère plus que les pauvres et, en dépit de leur égoïsme et de leur rapacité naturelle, quoiqu'ils n'aspirent qu'à leur propre commodité, quoique

1 Traduites pour notre propos: la coordination est-elle possible? Aboutit-elle à une seule solution? et celle-ci peut-elle être atteinte?

2 Nous rappelons qu'il y a trois occurrences du syntagme 'main invisible' dans l'œuvre de Adam Smith. (Smith 1976b, IV ii 9; 1976a, IV 1.10; 1980, History of Astronomy III 2). 
l'unique fin qu'ils se proposent d'obtenir du labeur des milliers de bras qu'ils emploient
soit leur seule satisfaction de leurs vains et insatiables désirs, ils partagent tout de même
avec les pauvres les produits des améliorations qu'ils réalisent. Ils sont conduits par une
main invisible à accomplir presque la même distribution des nécessités de la vie que celle
qui aurait eu lieu si la terre avait été divisée en proportions égales entre tous ses habi-
tants; et, ainsi, sans le vouloir, sans le savoir, ils servent les intérêts de la société et
donnent des moyens à la multiplication de l'espèce. Quand la providence partagea la
terre entre un petit nombre de grands seigneurs, elle n'oublia ni n'abandonna ceux qui
semblaient avoir été négligés dans la répartition. (Smith 1999, IV 1.10, pp. 257-258)

Sans vouloir faire une analyse précise du texte ni de ses différentes interprétations, notamment celle très idéologique et presque gratuite de Milton Friedman (1980, p. 32), nous relevons que Adam Smith soutient que le désir de richesse ne servira probablement pas l'intérêt à long terme de l'individu. La Nature nous joue des tours à notre insu et il est bien qu'il en soit ainsi: «Il est heureux que la nature nous abuse de cette manière. C'est cette illusion [les plaisirs de la richesse et de la grandeur] qui suscite et entretient le mouvement perpétuel de l'industrie du genre humain» (Smith 1999, IV 1.10, p. 256).

Il y a un sens transcendent, qui n'est pas en possession des individus, qui sont trompés par la Nature, mais il existe et il s'actualise grâce à la main invisible qui conduit les actions des hommes, comme la main qui place les pièces sur un échiquier, pour utiliser une autre métaphore de Smith (1999, VI 2.2, p. 324). Cependant, ce sens est saisissable et il est saisi par la raison tandis que la main invisible vient à constituer la relation entre le général et le particulier, le collectif et l'individuel.

\section{...MAIS QUI NE PEUT PLUS L'ÊTRE}

\section{Connaissance des mécanismes de coordination}

Revenons à «ces systèmes qui fonctionnent de manière aussi aveugle que les choses.» Dans le texte de Kafka, le paysan est animé par la volonté d'accéder à la loi, à la règle dont la compréhension lui échappe mais qui le concerne pourtant directement.

Deux éléments semblent ainsi être incontournables: la coordination des choix et la compréhension des mécanismes de cette coordination.

Si le premier aspect, celui de la coordination des décisions, est peut-être même constitutif de l'économie politique, le deuxième, la connaissance de ces mécanismes, est devenu centrale dans le dernier quart de siècle. La connaissance du fonctionnement de l'économie est essentiel pour l'agent économique dans la mesure où il est contraint de faire des prévisions sur la valeur future d'un certain nombre de paramètres. La qualité de ces choix actuels dépend ainsi de la qualité de ses prévisions sur le futur.

Or, cette question des croyances des agents économiques concernant la valeur future des paramètres pertinents a trouvé une solution très ingénieuse avec les anticipations rationnelles ${ }^{3}$.

Pour une contextualisation assez accessible, nous renvoyons à Snowdon, Vane et Wynarczyk (1994, ch. 5). 
C'est John F. Muth (1961) qui lance l'idée de l'agent anticipateur qui se base sur les annonces formulées par les autres acteurs économiques (gouvernement et institutions monétaires incluses). Dans son article, Muth met en valeur l'importance de la connaissance du modèle sous-jacent par tout acteur économique, l'hypothèse d'information parfaite est toujours présente. Mais c'est seulement avec Robert E. Lucas dans les années 1970 que la théorie des anticipations rationnelles devient le principal moteur des modèles économiques.

Il s'agit à cette époque de formuler une hypothèse forte quant à la façon de déterminer la vision de l'agent économique par rapport à l'avenir et surtout comment leurs attentes modifieraient le résultat de l'économie dans son ensemble à chaque période successive.

\begin{abstract}
The aim of the research is to build models that can predict how people's behavior will change when they are confronted with well-understood changes in ways of administering taxes, government purchases, aspects of monetary policy, and the like. (Sargent 1986, pp. 3-4)
\end{abstract}

Les anticipations rationnelles sont alors définies comme la 'meilleure supposition sur l'avenir' en utilisant toute l'information disponible. Il s'agit donc, encore une fois, de connaître le comportement des agents économiques (information parfaite) et de savoir utiliser cette information de façon à en tirer l'utilité maximale possible (rationalité économique). Cette façon de procéder ne tient pas compte du passé de la même façon que la théorie des anticipations adaptatives, qui prévoit les comportements des agents (ou la valeur des agrégats) en se basant sur leur histoire. La critique formulée par les théoriciens des anticipations rationnelles aux anticipations adaptatives consiste à dire que, ces dernières n'étant qu'une extrapolation du passé, le simple réflexe des équilibres antérieurs, ne sont pas en mesure de prévoir l'équilibre futur. Au contraire, les anticipations rationnelles considèrent explicitement la capacité de l'agent rationnel à prendre en compte les mécanismes économiques pour générer des prévisions. Ainsi, les anticipations sont dites rationnelles puisqu'elles sont générées par le modèle de l'économie qui est, a posteriori, le 'vrai modèle de l'économie' (c'est-à-dire que les variables de ce modèle prennent la valeur prévue, à un facteur aléatoire près).

La notion d'équilibre retenue dans ce contexte revient à affirmer, entre autre, que les agents ne sont pas amenés à revoir leurs plans. Les anticipations rationnelles ne sont pas forcément exactes, mais l'erreur ne peut pas être systématique car si elle l'était, les agents rationnels s'en apercevraient. Ils en seraient insatisfaits et l'intégreraient dans leurs calculs : ils changeraient ainsi leurs plans, ce qui est incompatible avec la définition même de l'équilibre.

Pour que les anticipations rationnelles existent et puissent être utilisées, l'équilibre est (usuellement) postulé en tout temps. Mais si l'équilibre est permanent, cela implique que la coordination l'est aussi: elle n'est plus un problème car elle est automatiquement réalisée ... par hypothèse !

Les anticipations rationnelles ont aussi une autre implication assez importante. Pour opérer un choix rationnel, celui qui choisit doit disposer d'un modèle du fonctionnement de l'économie. Mais cette représentation antérieure relève des anticipations rationnelles seulement dans la mesure où elle occasionne des choix qui provoquent l'état du monde qui lui est conforme. Finalement, ces choix ainsi pensés sont, une fois agis, confirmés. Les anticipations rationnelles impliquent 
alors leur autoréalisation. Les agents rationnels ont donc un comportement semblable, ou même identique. Il n'est pas alors surprenant, dans ce cadre théorique, qu'on recourt désormais de plus en plus à l'agent représentatif.

Dans la théorisation économique contemporaine, tout au moins celle qui fait référence aux anticipations rationnelles (la nouvelle macroéconomie, la finance,...), le fonctionnement est connu (et l'acteur rationnel): il n'y a plus d'espace que pour des comportements semblables, forcément autoréalisateurs, dans une séquence continue d'équilibres. Finalement, par rapport à ce schéma conceptuel, la question même de la coordination ne peut plus se poser.

On peut donc difficilement considérer qu'il y a «progrès» dans la réflexion théorique
lorsqu'on évacue «par hypothèse» ce qui devrait être l'objet même de cette réflexion. Il
est clair, de ce point de vue, que le fait de postuler des anticipations rationnelles constitue
une régression intellectuelle (comme l'est le recours à des agents représentatifs), même
si l'utilisation de techniques mathématico-statistiques complexes peut faire croire le
contraire au non-initié. (Guerrien 2002, p. 31)

La question de la coordination ne se pose plus, ne peut plus se poser, mais cela n'implique pas qu'il n'y a plus de coordination dans les modèles macroéconomiques. Bien au contraire, il y en a plus que jamais: au même titre que l'équilibre, la coordination est simplement hypostasiée, c'est-à-dire qu'il s'agit d'une pure abstraction qui est considérée abusivement comme une réalité. Cela signifie qu'en l'état actuel, la seule façon que les économistes ont trouvée pour représenter des agents qui choisissent rationnellement à l'aide d'une représentation du fonctionnement de l'économie, est de considérer le système constamment à équilibre, ce qui implique de renoncer à problématiser la convergence vers l'équilibre, c'est-àdire la coordination même.

Le paysan de la nouvelle de Kafka ne sait pas qu'il ne connaîtra pas le fonctionnement: il ne le saura qu'après coup. L'agent rationnel ne sait pas si le modèle de fonctionnement qu'il connaît est le bon: il ne le saura qu'après coup. Le modèle fonctionne comme médiateur, incertain mais complaisant, entre le sujet et son action; au contraire le gardien, certain et frustrant, empêche la médiation. Mais le modèle ne permet plus la quête du sens, alors que le gardien permet (oblige) cette quête.

Certes, la rationalité instrumentale, outil par excellence et presque identitaire de l'économiste, sous-jacente aux anticipations rationnelles, peut probablement porter à elle seule la responsabilité pour cette impossibilité. Cependant, la réflexion sur les anticipations rationnelles nous pose la question différemment: la connaissance des mécanismes n'exclut-elle pas la quête de sens, qui, en fait, se trouverait plutôt situé entre l'impensable et le savoir.

Finalement, est-ce que l'attente du paysan ne serait finalement pas semblable à celle d'Estragon et de Vladimir ${ }^{4}$, pour qui la quête n'a de sens qu'en elle-même?

\section{Université de Lausanne}

${ }^{4}$ En attendant Godot, tragicomédie en deux actes de Samuel Beckett (1952). 


\section{RÉFÉRENCES BIBLIOGRAPHIQUES}

Beckett, Samuel. 1952. En attendant Godot. Paris: Les éditions de minuit.

Friedman, Milton et Rose Friedman. 1980. Free to Chose. A personal Statement. New York: Penguin Books.

Guerrien, Bernard. 2002. Dictionnaire d'analyse économique: microéconomie, macroéconomie, théorie des jeux, etc. Paris: La Découverte.

Muth, John F. 1961. «Rational Expectations and the Theory of Price Movements.» Econometrica, 29:3, pp. 315-335.

Sargent, Thomas J. 1986. Rational Expectations and Inflation. New York: Harper \& Row.

Smith, Adam. 1976a. The Theory of Moral Sentiments. The Glasgow edition of the works and correspondence of Adam Smith: vol. I. D.D. Raphael et A.L. Macfie éds. Oxford: Clarendon Press.

- 1976b. An Inquiry into the Nature and Causes of the Wealth of Nations. The Glasgow Edition of the Works and Correspondence of Adam Smith: vol. II. R.H. Campbell et A.S. Skinner éds. Oxford: Claredon Press.

- 1980. Essays on Philosophical Subjects. The Glasgow edition of the works and correspondence of Adam Smith: vol. III. W.P.D. Wightman, J.C. Bryce et I.S. Ross éds. Oxford: Clarendon Press.

- 1999. Théorie des sentiments moraux. Paris: Presses Universitaires de France.

Snowdon, Brian, Haward Vane et Peter Wynarczyk. 1994. A Modern Guide to macroeconomics. An Introduction to Competing Schools of Thought. Aldershot: Edward Elgar.

Walras, Léon. 1988. Eléments d'économie politique pure. Auguste et Léon Walras œuvres économiques complètes: vol. VIII. C. Mouchot éd. Paris: Economica. 